\title{
Solar flares observed by AVS-F instrument onboard CORONAS-F satellite during 2,5 year of it's operation
}

\author{
Andrew Arkhangelsky ${ }^{1}$, I. V. Arkhangelskaja ${ }^{1}$, Yu. D. Kotov ${ }^{1}$, \\ A.S Glyaneneko and S. N. Kuznetsov ${ }^{2}$ \\ ${ }^{1}$ Astrophysics Institute, Moscow State Engineering Physics Institute (State University) \\ Kashirskoe shosse 31, Moscow, 115409 Russia email: angel966@list.ru \\ ${ }^{2}$ Scobeltsyn Institute of Nuclear Physics, Moscow State University, 119899 Russia \\ email:kuznets@srd.sinp.msu.ru
}

\begin{abstract}
AVS-F instrument (Amplitude-Time Spectrometry of the Sun) is the system of an electronics engineering for onboard data gathering from two detectors: scintillation (CsI (Tl)) detector SONG-D (SOlar Neutrons and Gamma quantums) of complex of detectors SKL (in low and high gamma-band) and from semiconducting detector (X-ray semiconducting spectrometer) XSS-1 (in X-ray band). The experiment is carried out on the satellite CORONAS-F launched on July 31 2001. During more than 2,5 year of apparatus operation more than 30 of solar flares were detected. Characteristics of observed solar flares are presented in this article.
\end{abstract}

The AVS-F (amplitude-time Sun spectrometry) instrumentation (description see Arkhangelsky et al (1999))is intended to study characteristics of fluxes of hard X-rays, $\gamma$-rays and neutrons from the Sun and solar flares and to detect and record events like $\gamma$-ray bursts (non-stationary fluxes of $\gamma$-rays from space). The experiment is conducted under the CORONAS (Complex ORbiting ObservatioNs of the Active Sun) international project and carry out onboard CORONAS-F special-purpose automatic station (NORAD catalogue number 26873, International Designator 2001-032A) that had been launched from Russian kosmodrom Plesetsk at 11:00 UT of 31 July 2001 into a circular orbit oriented towards the Sun with inclination $82.5^{\circ}$ and altitude $\sim 500 \mathrm{~km}$.

A function diagram of the AVS-F instrumentation is shown in Figure 1.a. The instrumentation consists of two detectors and AVS-F hardware unit. The AVS-F apparatus use signals produced by the SONG-D detector (energy deposition ranges of $0.1 \div 17.0 \mathrm{MeV}$ and $4.0 \div 94.0 \mathrm{MeV}$ by up to date calibration data (Arkhangelskaja et al (2002)) based on the $\mathrm{CsI}(\mathrm{Tl})$ crystal $\varnothing 20 \mathrm{~cm}$ and height of $10 \mathrm{~cm}$ with electronics unit SONG-E (Devicheva et al (1999)) for $\gamma$-rays and neutron measurements, semiconductor detector $\mathrm{XSS}-1(3 \div 30 \mathrm{keV}$ energy deposition range) and the $\mathrm{AC}$ is the anticoincidence signal generated by the plastic scintillation counter of the SONG-D.

Above 30 solar flares with class M2 and higher in GOES classification were registered during 2.5 year of AVS-F device operation. It was observed 14 flares with class $\mathrm{X}$ and 9 flares with class higher than M5.0 (see Table 1). It is seen that in most part of flares cases gamma-emission interval is shorter than X-ray one. The examples of solar flares temporal profiles are shown in Figures 1.b,c. At present (at the moment of writing this article), the AVS-F instrument successfully operates onboard CORONAS-F satellite during period above 2.5 year. Total amount of the received information is about $6.5 \mathrm{~GB}$. The apparatus works from launching up today's without any malfunctions accordingly to telemetry test information. 
Table 1. The characteristics of solar flares with class > M5.0 observed by AVS-F

day

25.08.2001

05.09 .2001

19.10.2001

19.10.2001

19.10.2001

23:10:2001

04.11 .2001

07.11 .2001

25.11.2001

04.04 .2002

21.04 .2002

11.07 .2002

17.07 .2002

20.07 .2002

26.07 .2002

24.08 .2002

17.03 .2003

11.06 .2003

26.10 .2003

29.10 .2003

04.11 .2003
AVS-F time*

16:28:06-16:38:44

14:27:06-14:30:01

01:20:45-01:37:01

09:33:54-09:38:26

16:15:00-ERB

02:15:16-02:16:52

16:06:00-16:14:00

19:33:03-19:34:40

BTG-09:56:38

15:28:53-15:31:03

BTG,BRB - 02:33:00

14:47:24-14:49:49

07:11:11-07:14:39

TG, $21: 27: 49-21: 35: 25, \mathrm{ERB}$

22:06:22-22:09:57,ESH

00:57:00-01:06:16,ERB

18:57:35-ERB

20:06:15-20:08:54

$21: 35: 20-21: 37: 56$

20:40:00-21:01:00

class active region coord. GOES time

X5.3 $9591 \quad$ S17E34 16:23-17:04

$\begin{array}{llll}\text { M6.0 } & 9601 \quad \text { N15W31 } & 14: 25-14: 34\end{array}$

X1.6 $9661 \quad$ N16W18 00:47-01:13

$\begin{array}{llll}\text { M5.7 } & 9658 & \text { S14W47 } & 09: 35-09: 55\end{array}$

X1.6 $9661 \quad$ N15W29 16:13-16:43

M6.5 $\quad 9672 \quad$ S18E11 02:11-02:34

$\begin{array}{llll}\mathrm{X} 1.0 & 9684 & \text { N06W18 } & 16: 03-16: 57\end{array}$

M5.7 $\quad 9690 \quad$ S17E44 19:30-20:26

$\mathrm{X} 1.1$

M6.1

$\mathrm{X} 1.5$

M5.8

M8.5

$\mathrm{X} 3.3$

M5.3

$\mathrm{X} 3.1$

$\mathrm{X} 1.5$

$\mathrm{X} 1.6$

M7.6

$\mathrm{X} 10.0$

19:33:01-19:34:00; 19:37:35-20:23:25 X17.4

09:45-09:54

15:24-15:38

$9906 \quad$ S14W84 00:43-02:38

$0030 \quad$ N21E58 14:44-14:57

$0030 \quad$ N21W17 06:58-07:19

21:04-21:54

22:03-22:32

$0069 \quad$ S02W81 00:49-01:31

0314 S14W39 18:50-19:16

0375 N14W57 20:01-20:27

$0484 \quad$ N01W40 21:34-21:48

0486 S19W09 20:37-21:01

0486 S18W88 19:29-20:06

* BRB (ERB) - flare's begin (end) in radiation belt, ESH - flare's end in the Earth shadow, BTG - flare's begin in telemetry gap

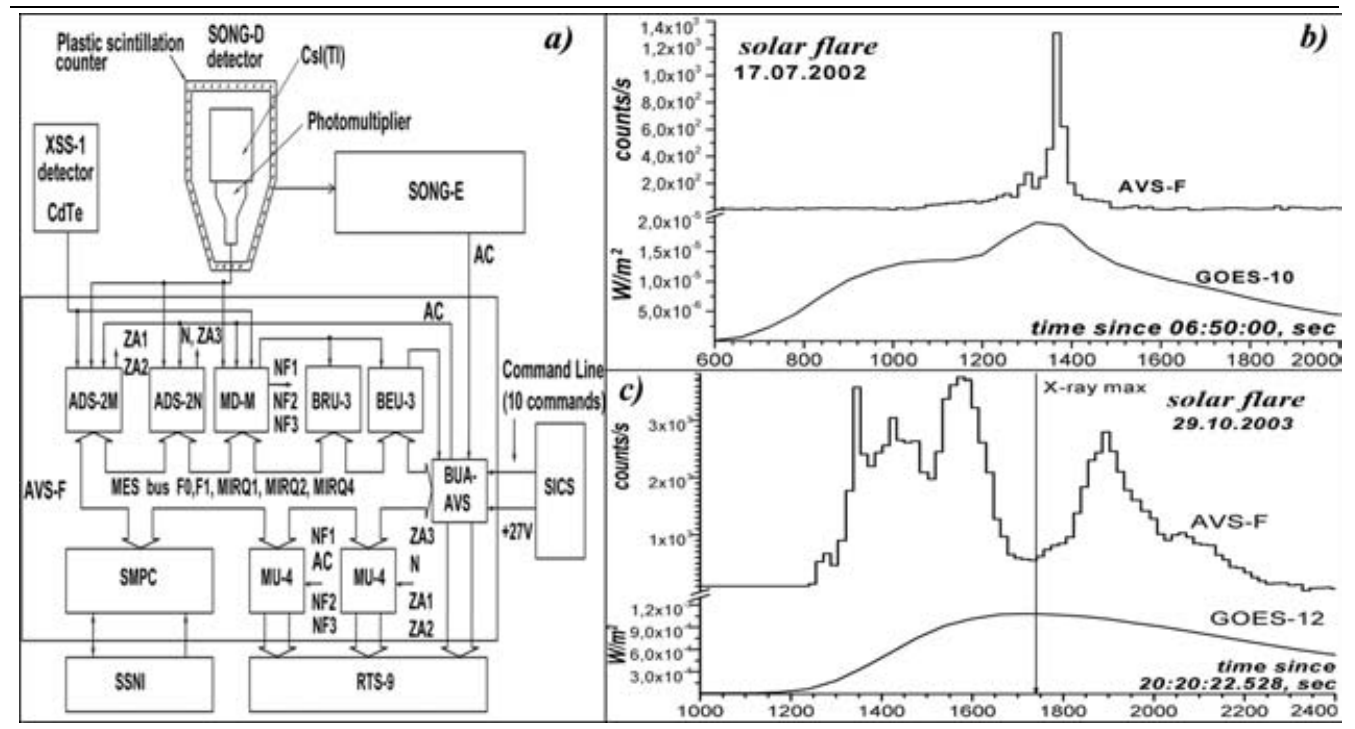

Figure 1. The functional diagram of AVS-F apparatus (a) and examples of solar flares temporal profile on AVS-F and GOES data $(b, c)$

\section{References}

Arkhangelskaja, I. V., Arkhangelky, A. I., Glyanenko, A. S. et al 2002 Bulletin of the Russian Academy of Sciences: Physics $\mathbf{6 7} N^{o} \mathbf{1 1}, 1621-1623$.

Arkhangelsky, A.I., Glyanenko, A. S., Kotov Yu. D., et al. 1999 IET 42 N ${ }^{o} \mathbf{5}, 596-603$.

Devicheva, E. A., Dobrovolskii, G. F., Kovalevskaya, M. A. et al. 1989 In Comprehensive Studies of the Sun and Solar-Earth Links, pp. 99-110. LFTI, Leningrad, Russia. 